\title{
Evaluation of a novel Y-shaped extracardiac Fontan baffle using computational fluid dynamics
}

\author{
Alison L. Marsden, PhD, ${ }^{a}$ Adam J. Bernstein, BSE, ${ }^{\mathrm{b}}$ V. Mohan Reddy, MD, ${ }^{\mathrm{c}}$ Shawn C. Shadden, PhD, ${ }^{\mathrm{d}}$ Ryan L. Spilker, MS, \\ Frandics P. Chan, MD, PhD, ${ }^{f}$ Charles A. Taylor, $\mathrm{PhD},{ }^{\mathrm{d}, \mathrm{g}}$ and Jeffrey A. Feinstein, MD, $\mathrm{PhD}^{\mathrm{d}, \mathrm{g}}$
}

\begin{abstract}
Objectives: The objective of this work is to evaluate the hemodynamic performance of a new Y-graft modification of the extracardiac conduit Fontan operation. The performance of the Y-graft design is compared to two designs used in current practice: a t-junction connection of the venae cavae and an offset between the inferior and superior venae cavae.
\end{abstract}

\begin{abstract}
Methods: The proposed design replaces the current tube grafts used to connect the inferior vena cava to the pulmonary arteries with a Y-shaped graft. Y-graft hemodynamics were evaluated at rest and during exercise with a patient-specific model from magnetic resonance imaging data together with computational fluid dynamics. Four clinically motivated performance measures were examined: Fontan pressures, energy efficiency, inferior vena cava flow distribution, and wall shear stress. Two variants of the Y-graft were evaluated: an "off-the-shelf" graft with 9-mm branches and an "area-preserving" graft with 12-mm branches.
\end{abstract}

Results: Energy efficiency of the 12-mm Y-graft was higher than all other models at rest and during exercise, and the reduction in efficiency from rest to exercise was improved by $38 \%$. Both Y-graft designs reduced superior vena cava pressures during exercise by as much as $5 \mathrm{~mm} \mathrm{Hg}$. The Y-graft more equally distributed the inferior vena cava flow to both lungs, whereas the offset design skewed $70 \%$ of the flow to the left lung. The 12-mm graft resulted in slightly larger regions of low wall shear stress than other models; however, minimum shear stress values were similar.

Conclusions: The area-preserving 12-mm Y-graft is a promising modification of the Fontan procedure that should be clinically evaluated. Further work is needed to correlate our performance metrics with clinical outcomes, including exercise intolerance, incidence of protein-losing enteropathy, and thrombus formation.

Supplemental material is available online.

Computational modeling, clinical observation, and previous experimental work suggest the geometry of the total cavopulmonary connection (TCPC; Fontan) plays a key role in energy losses, ${ }^{1-8}$ that is, efficiency, and Fontan outcomes. Previous simulation-derived alternatives led to the adoption of an offset of the inferior anastomosis relative to the superior anastomosis and reduced energy losses when compared

From the Mechanical and Aerospace Engineering Department, University of California, San Diego, ${ }^{\text {a }}$ and the Electrical Engineering Department, ${ }^{\text {b }}$ Department of Cardiothoracic Surgery, ${ }^{\mathrm{c}}$ Bioengineering Department, ${ }^{\mathrm{d}}$ Mechanical Engineering Department, ${ }^{\mathrm{e}}$ Department of Radiology ${ }^{\mathrm{f}}$ and Department of Pediatrics, ${ }^{\mathrm{g}}$ Stanford University, Stanford, Calif.

This work was supported by the National Science Foundation under grant No. 0205741 and the Vera Moulton Wall Center for Pulmonary Vascular Disease at Stanford University. Dr. Marsden was supported by a postdoctoral fellowship from the American Heart Association and a Burroughs Wellcome Fund Career Award at the Scientific Interface.

Received for publication Nov 9, 2007; revisions received April 1, 2008; accepted for publication June 15, 2008.

Address for reprints: Alison L. Marsden, PhD, Mechanical and Aerospace Engineering, University of California San Diego, 9500 Gilman Drive, La Jolla, CA (E-mail: amarsden@stanford.edu).

J Thorac Cardiovasc Surg 2009;137:394-403

$0022-5223 / \$ 36.00$

Copyright (c) 2009 by The American Association for Thoracic Surgery doi:10.1016/j.jtcvs.2008.06.043 with the traditional t-shaped junction. ${ }^{3,9,10}$ Recent work has also examined the effects of exercise on energy loss in multiple patient-specific models. ${ }^{11,12}$

Recently, and concurrently with our work, Soerensen and associates ${ }^{13}$ proposed a similar design called the "OptiFlo", in which the native inferior (IVC) and superior venae cavae (SVC) are bifurcated before the pulmonary artery anastomosis. Their study demonstrated reduced power loss at rest and simulated exercise with simplified geometric models and steady inflow conditions. The two studies share the concept of eliminating flow competition by bifurcating the graft used in the extracardiac Fontan procedure. However, our study addresses some of the computational limitations of this previous study through the use of more detailed geometric models, pulsatile inflow conditions, and sophisticated outflow boundary conditions. In addition, our proposed Y-graft design offers several important technical advantages: it can be easily modified for an individual patient, it can be custom manufactured of synthetic material using realistic sizes, and it allows the procedure to be performed without cardiopulmonary bypass.

$\mathrm{We}^{11}$ have previously reported the development of an increasingly accurate Fontan modeling system, producing realistic pressure and flow data when compared with that measured by catheterization and echocardiography. This was accomplished by incorporating increased anatomic accuracy, specifically a greater level of pulmonary branching, 

Abbreviations and Acronyms
IVC = inferior vena cava
LPA = left pulmonary artery
MRI = magnetic resonance imaging
$\mathrm{PC}=$ phase-contrast
$\mathrm{RPA}=$ right pulmonary artery
SVC = superior vena cava
TCPC $=$ total cavopulmonary connection
WSS $=$ wall shear stress

\section{Image/Anatomy Acquisition}

Magnetic resonance angiography was performed with a 1.5-T magnetic resonance imaging (MRI) scanner (Signa TwinSpeed; General Electrics, Milwaukee, Wis). During intravenous administration of a gadolinium-based contrast agent, images were acquired using a half-Fourier, 3-dimensional fast gradient-recalled echo sequence with breath-holding. The spatial resolution was approximately $0.7 \mathrm{~mm} \times 1.2 \mathrm{~mm} \times 2.0 \mathrm{~mm}$. Flow waveforms in the venae cavae were recorded with a 2-dimensional phase-contrast (PC) MRI method. The imaging plane was placed perpendicular to the dominant flow with velocity encoding parallel to the flow. The slice thickness was 10 $\mathrm{mm}$ and the in-plane resolution was about $1.0 \mathrm{~mm} \times 1.7 \mathrm{~mm}$. The encoding velocity was $120 \mathrm{~cm} / \mathrm{s}$. Velocity data were acquired over several cycles of free breathing with the use of cardiac gating and respiratory compensation.

\section{Model Construction}

Once the image data have been acquired, there are four steps necessary to construct geometric models from image data volumes (Figure 1) ${ }^{19}$ (1) Centerline paths are created in the vessels of interest, (2) segmentations of the vessel lumen are created perpendicular to the centerlines using a 2-dimensional level set method, (3) the 2-dimensional segmentations are lofted together, creating a solid model of the desired vasculature, and (4) the solid model is discretized into an unstructured tetrahedron mesh for use in the finite element flow solver.

For this study, one patient-specific anatomy and three geometric variations were constructed. The first model was constructed from patient-specific MRI data from a 4-year-old girl who had a traditional extracardiac Fontan with the IVC and SVC anastomosed to the pulmonary arteries in the classic "t-junction" configuration (that is, with no offset, Figure 2). Subsequently, by use of the same custom model construction software, three variations (left pulmonary artery [LPA] offset, small-Y, large-Y, Figure 2) on the initial, patient-specific design were created by changing the geometry of the IVC connection while keeping the SVC and pulmonary geometries identical. In this way, the effect of the IVC connection geometry on hemodynamic performance could be examined independently and compared with the original $t$-junction design. The pulmonary vasculature was constructed to include all pulmonary branches larger than or equal to the segmental branches, representing the resolution limits of the MRI data for this patient.

In the offset model, the IVC was offset approximately one IVC diameter from the SVC toward the LPA to represent current clinical practice. Two variations of the Y-graft were constructed.

The first (small-Y) was constructed with an 18-mm trunk with two 9-mm branches, modeled after grafts that are approved by the Food and Drug Administration and currently available. The second (large-Y) was designed to approximately preserve the cross-sectional area between the trunk (18 $\mathrm{mm})$ and two branches (each $12 \mathrm{~mm}$ ). While an exact area preservation would result in 12.7-mm branches, the smaller size was chosen to achieve a better fit to the pulmonary arteries for this patient and because grafts are typically manufactured with standardized integer dimensions. The IVC trunk dimension of $18 \mathrm{~mm}$ was chosen in accordance with current practice at our institution. In a recent study of more than 300 patients, $97 \%$ received conduits of $18 \mathrm{~mm}$ or larger, and $69 \%$ received conduits of $20 \mathrm{~mm}$ or larger. ${ }^{9}$ These results also agree with previous studies determining optimal graft size. ${ }^{20}$

\section{Flow Simulations and Boundary Conditions}

To simulate blood flow, we used a custom stabilized finite element solver to solve the time-dependent, 3-dimensional Navier-Stokes equations. ${ }^{21,22}$ An anisotropic adaptive meshing scheme ${ }^{17}$ was used to ensure mesh convergence using approximately 1.5 million elements for each model. A newtonian approximation for the viscosity was assumed with a value of 0.04 $\mathrm{g} /(\mathrm{cm} \mathrm{s})$ and the density of blood was $1.06 \mathrm{~g} / \mathrm{cm}^{3}$. A rigid-wall approximation was used. Further details on our Fontan simulation methods and flow solver can be found in the article by Marsden and associates. ${ }^{11}$

Respiratory-varying boundary conditions were imposed at the IVC inlet in all simulations. Pulsatile flow rates were acquired from patient-specific 

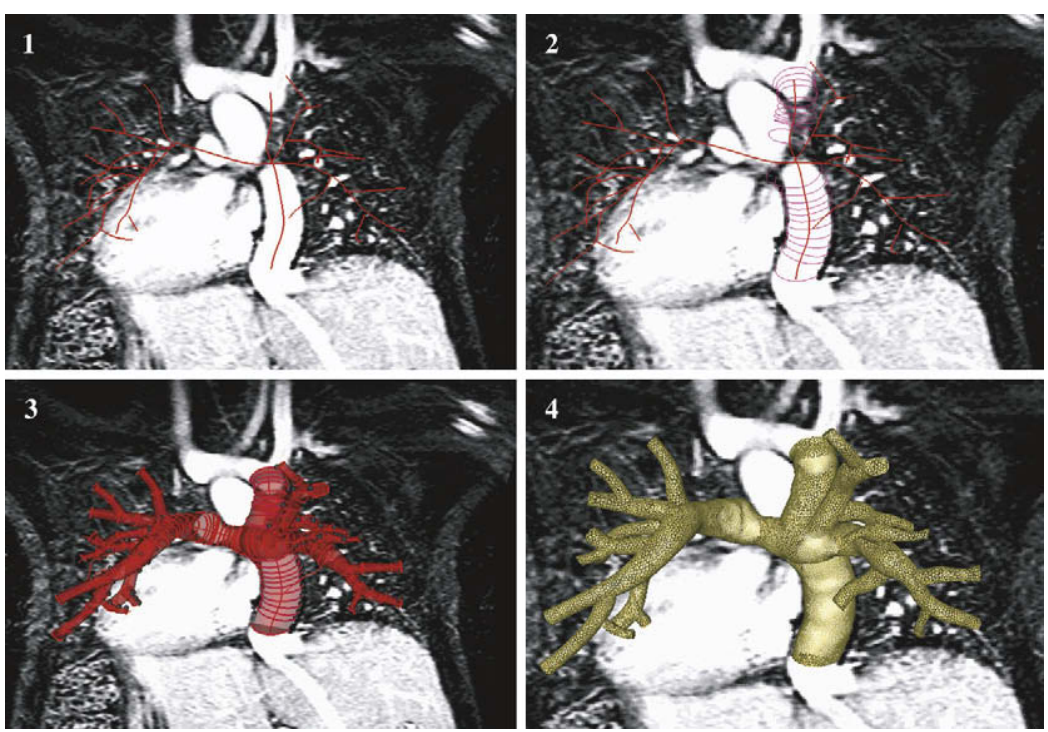

FIGURE 1. Four steps involved in model construction are (1) creation of vessel paths, (2) segmentation, (3) lofting, and (4) mesh generation. Image is displayed posterior to anterior for better viewing.

PC-MRI data and were superimposed on a respiration model to construct inflow waveforms. ${ }^{11}$ Since little respiratory variation is observed in the SVC, the PC-MRI flow waveform was imposed at the SVC inflow face with no respiration model. The IVC and SVC flow waveforms were mapped to a parabolic profile on the inlet faces of the model.

Four flow rate levels were used in the IVC: normal/resting (ie, measured) flow and 2,3, and 4 times normal flow to simulate light, moderate, and heavy exercise conditions. Although the heavy exercise condition may not be physically attainable by most Fontan patients, simulations allow us to push the limits of performance for evaluation purposes. Flow rates in the SVC were increased only for the highest level of exercise, by $50 \%{ }^{23}$
Table 1 lists the mean rest and exercise flow rates used in simulations for all models.

Initial downstream resistance values were calculated to match patientspecific cardiac catheterization pressure data $(7 \mathrm{~mm} \mathrm{Hg}$ in the IVC and SVC). An overall flow split of $45 \%$ to the LPA and $55 \%$ to the right pulmonary artery (RPA) was assumed. On each side, flow was distributed among the pulmonary outlets by grouping them into upper, middle, and lower lobes. Initial resistances were chosen to distribute $20 \%$ of the flow to the upper lobe and $40 \%$ each to the middle and lower lobes. The LPA and RPA branches of the model were then grouped by lobe with 2 segments in the upper lobe and 4 segments in each of the lower and middle lobes on
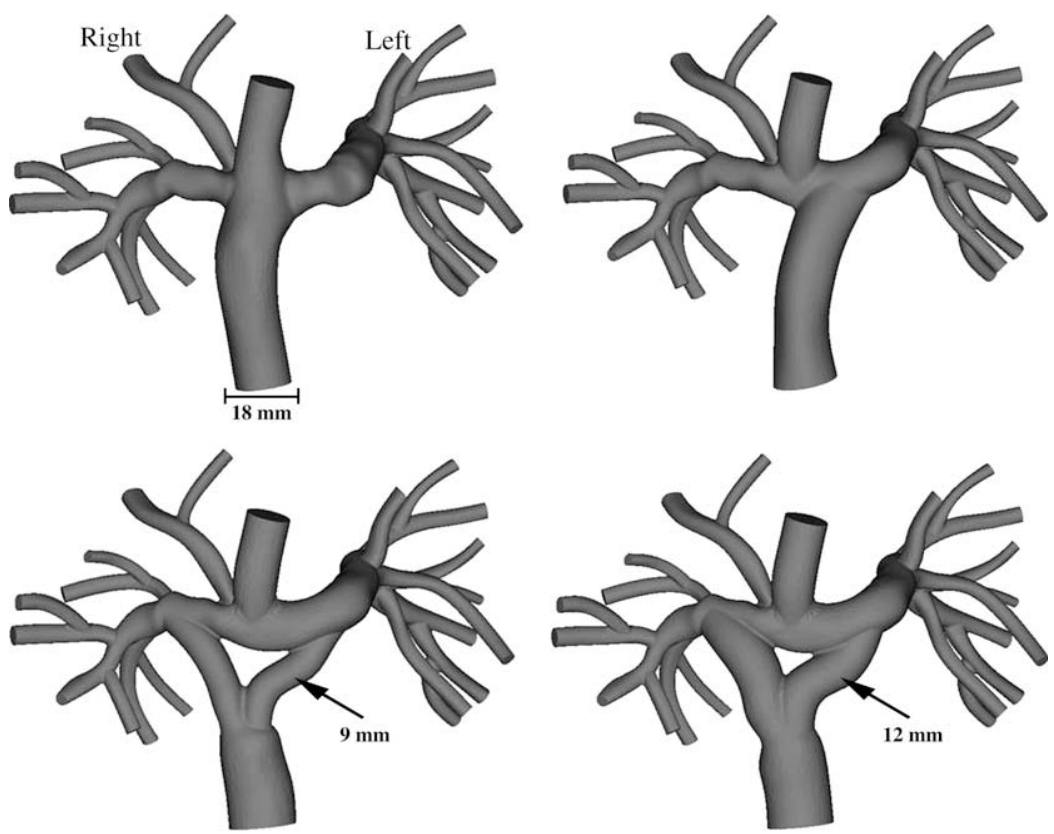

FIGURE 2. The four Fontan geometries: an MRI-derived, patient-specific t-shaped connection (top left), computer-aided design-derived LPA offset model (top right), Y-graft with 9-mm branches (lower left), and Y-graft with 12-mm branches (lower right). 
TABLE 1. Mean flow rates in the IVC and SVC and resistance reductions at rest and three levels of simulated exercise

\begin{tabular}{lccc}
\hline \multicolumn{1}{c}{ Exercise level } & IVC $(\mathbf{m L} / \mathbf{s})$ & SVC $(\mathbf{m L} / \mathbf{s})$ & Resistance drop $(\%)$ \\
\hline Rest & 17.9 & 14.1 & 0 \\
Light exercise & 35.8 & 14.1 & 5 \\
Moderate exercise & 53.7 & 14.1 & 10 \\
Heavy exercise & 71.6 & 21.2 & 15 \\
\hline
\end{tabular}

$I V C$, Inferior vena cava; $S V C$, superior vena cava.

each side. Within each lobe group, flow was distributed according to the outlet areas. By dilation of the morphometry-based arterial trees, the resistance values were decreased by $5 \%, 10 \%$, and $15 \%$ for the light, moderate, and heavy exercise simulations, respectively. ${ }^{24}$

A 3 -element windkessel model ${ }^{25}$ was assigned to each of the 20 outlets of the 3-dimensional model using the coupled multidomain method. ${ }^{14}$ The resistance and capacitance parameters were chosen so that the impedance of the windkessel model best fit the impedance of a morphometry-based pulmonary arterial tree constructed at each outlet of the Fontan model. ${ }^{15}$ At a given level of exercise, identical boundary conditions were imposed for all models considered in this study.

Each simulation was run on 20 to 24 processors of an SGI Altix parallel supercomputer (SGI, Sunnyvale, Calif), requiring about 1000 CPU hours (2 days) of run time. Simulation results were post-processed to interpret velocity, pressure, and WSS information throughout the model.

\section{Energy Efficiency}

Efficiency was calculated for each model at rest and all exercise levels by calculating the rate of energy passing through all inlets and outlets using the equations shown below. Since all models shared identical pulmonary branches as well as identical inflow and outflow boundary conditions, changes in efficiency result only from changes in Fontan geometry.

The energy dissipation (neglecting gravitational effects) is given by

$$
\begin{aligned}
\mathrm{E}_{\mathrm{diss}}= & -\sum_{\mathrm{i}=1}^{\mathrm{N}_{\text {in }}} \int_{\mathrm{Ai}}\left(\mathrm{p}+\frac{1}{2} \rho \mathrm{u}^{2}\right) \mathbf{u} \cdot \mathrm{dA} \\
& -\sum_{\mathrm{i}=1}^{\mathrm{N}_{\text {out }}} \int_{\mathrm{Ai}}\left(\mathrm{p}+\frac{1}{2} \rho \mathrm{u}^{2}\right) \mathbf{u} \cdot \mathrm{dA},
\end{aligned}
$$

where $\mathrm{u}$ is the velocity, $p$ is the pressure, $\rho$ is the density, $\mathrm{N}_{\text {in }}$ and $\mathrm{N}_{\text {out }}$ are the number of model inlets and outlets, respectively, and $A_{i}$ is the area of the $i^{\text {th }}$ inlet or outlet. ${ }^{11,26}$ The energy efficiency is then

$\mathrm{E}_{\text {effic }}=\mathrm{E}_{\text {out }} / \mathrm{E}_{\mathrm{in}}$,

where $E_{\text {in }}$ is the first term in equation (1) and $E_{\text {out }}$ is the second term. The efficiencies we report in this work are the mean values over one respiratory cycle. Convergence studies confirmed that results did not change using multiple respiratory cycles.

\section{IVC Flow Distribution}

Particle-tracking methods ${ }^{27}$ were used to quantify the distribution of IVC flow to the right and left lungs. The inlet of the IVC was uniformly seeded with 35,000 particles every $1 / 50$ th of the respiratory cycle for an entire cycle. The particles were advected by the flow (Figure E1) until approximately all particles were flushed from the computational domain, requiring about two respiratory cycles. Particles were assumed to be massless, and effects of diffusion were neglected owing to the short residence time of the particles in the computational domain. The advection scheme used a Runge-Kutta-
Fehlberg ${ }^{28}$ integration algorithm with a maximum error tolerance of $10^{-5}$ and a linear interpolation of the velocity data in space and time.

The proportion of IVC flow going to the left lung was quantified by summing the number of particles that exited the computational domain through outlets connected to the LPA. Likewise, the portion of IVC flow to the right lung was quantified by summing the number of particles that exited through outlets connected to the RPA. It was verified that the results were independent of the seeding density and rate and the integration error tolerance.

\section{RESULTS \\ Hemodynamics}

A comparison of velocity fields from simulations of moderate exercise in the t-junction, LPA offset, small-Y, and large-Y Fontan models is shown in Figure 3. Qualitatively, the level of flow unsteadiness is very different for each model, with the large-Y model appearing to produce the least amount of unsteadiness. Helical flow is observed in the LPA side of the $t-j$ unction and offset models that is not observed in the Y-graft models. Similar observations are made during resting flow conditions.

Contours of mean pressures at peak inspiration during moderate exercise for the same four models are shown in Figure 4. A comparison of IVC and SVC pressure levels between the models shows substantial differences. The SVC pressure is lower with both Y-graft designs compared with either the t-junction or the offset model. The large-Y model has lower IVC pressures than the small-Y because there is no area reduction at the graft bifurcation.

Unexpectedly, we also observe that both Y-graft models result in a "pressure blocking" effect for the SVC (Figure 4). In the original t-junction model, the SVC pressure must overcome flow competition from the IVC. However, in both Y-graft models the SVC pressure is lower than the IVC pressure because the Y-graft geometry physically separates IVC and SVC flow.

Results from the $t$-junction simulations at rest verify that the pressure drop from the Fontan baffle to the LPA and RPA $(<1$ $\mathrm{mm} \mathrm{Hg}$ ) match the finding of "no significant pressure drops" reported during catheterization. Figure 5 quantifies the mean pressure levels in the IVC and SVC at rest and all levels of exercise for the four models. Pressures in the IVC were lowest with the large-Y model, although pressures in the offset design were only slightly higher. The small-Y IVC pressures were nearly as high as the t-junction Fontan. SVC pressures were substantially lower in both Y-graft models than in either the $\mathrm{t}$-junction or the offset models. Differences in mean pressure among all models are small during rest but increase during exercise to nearly $5 \mathrm{~mm} \mathrm{Hg}$ in the IVC and $3 \mathrm{~mm} \mathrm{Hg}$ in the SVC. During moderate exercise, the differences in peak pressure between the t-junction and the large $\mathrm{Y}$-graft are nearly $8 \mathrm{~mm} \mathrm{Hg}$ in the SVC and $4 \mathrm{~mm} \mathrm{Hg}$ in the IVC.

\section{Energy Efficiency}

The performance of the different models is quantified by examining the energy efficiency. Inasmuch as flow 

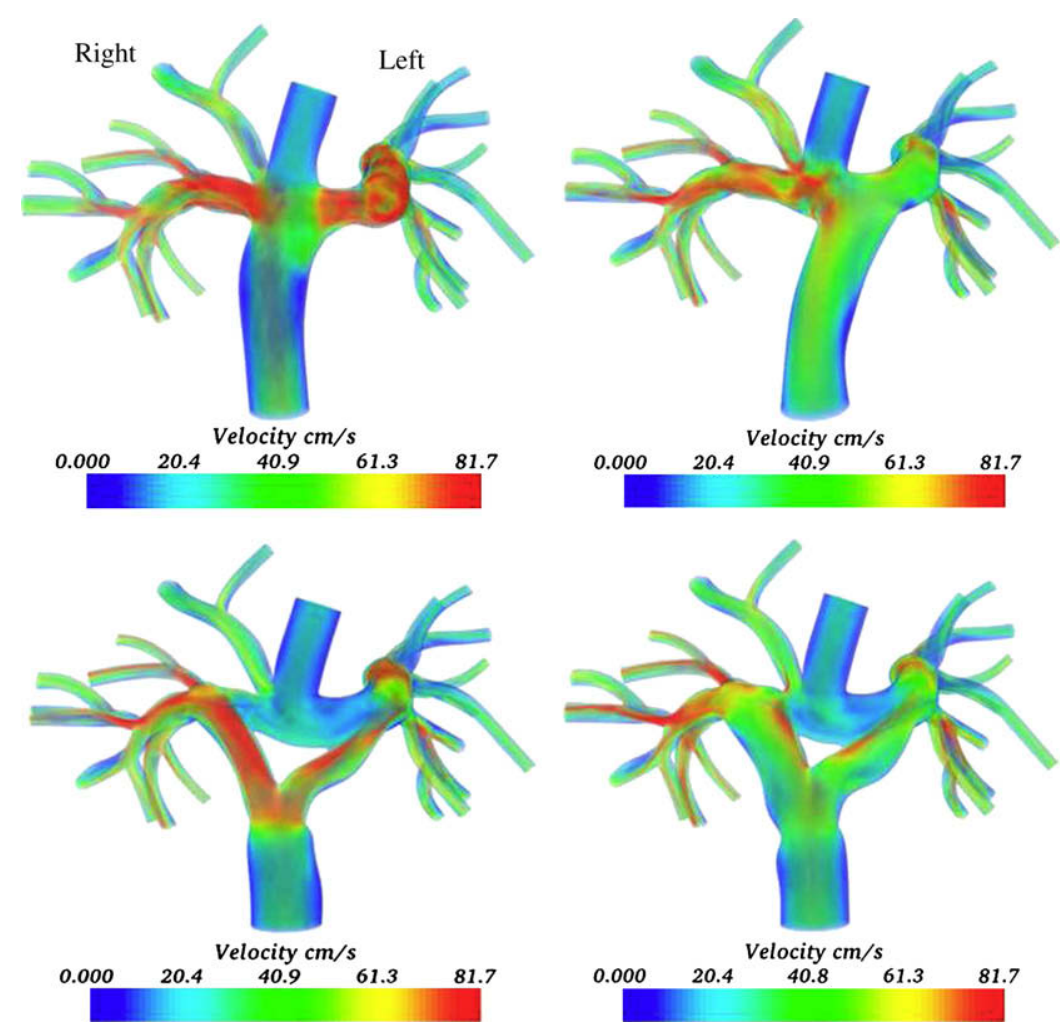

FIGURE 3. Volume rendered velocity magnitude from moderate exercise simulations. The Y-graft design results in reduced unsteadiness in the flow, especially during exercise. Velocities shown correspond to the peak of inspiration when velocity is maximum.

unsteadiness increases energy dissipation, a reduction in unsteadiness is reflected in higher energy efficiency values. Figure 6 compares mean efficiencies of the t-junction, LPA offset, small-Y, and large-Y models.

The t-junction Fontan had the lowest efficiency of all the models $(86.9 \%$ at rest, $75.8 \%$ at heavy exercise) and an efficiency reduction of $11.1 \%$. The reduction in efficiency with exercise was less severe for the offset and Y-graft models. The large-Y model had the highest efficiency of all models at rest $(90.3 \%)$ and all exercise levels and a rest/exercise reduction of only $6.9 \%$, a $38 \%$ improvement over the t-junction.

\section{IVC Flow Distribution}

Another presumed important determinant of performance is the relative distribution of IVC flow to the right and left lungs. The percentages of IVC flow going to the LPA and RPA for all simulations are given in Table 2. While the total (IVC+SVC) $45 / 55$ flow split is very closely enforced by the outflow boundary conditions in all cases, the relative distribution of SVC and IVC flow changes dramatically with the Fontan geometry. In the offset model, the majority of IVC flow is directed to the lung on the side of the offset, whereas most SVC flow goes to the opposite lung. In contrast, the Y-graft design evenly distributes both the IVC and SVC flow. The t-junction slightly favors the LPA side owing to a small asymmetry in the junction geometry in this particular patient.

\section{WSS}

Figure E2 shows the mean WSS values (dynes/ $\mathrm{cm}^{2}$ ) for the LPA offset model and the large Y-graft model over a respiratory cycle during resting and moderate exercise flow conditions. Qualitatively, the large Y-graft results in larger gradients in shear stress and somewhat larger areas of low WSS compared with the offset model, with low shear stress generally located in areas of high curvature. As expected, these differences are less pronounced during simulated exercise. Minimum and mean (in space) WSS values on the graft (IVC) portion of each model are given in Table 3. All values are the average in time over one respiratory cycle. The large-Y model has the lowest minimum WSS value of all models, but the mean value of WSS on the graft is higher than either the t-junction or LPA offset model. The small-Y model has the highest overall WSS (minimum and mean) on the graft compared with all other models owing to higher velocity flow in the branches caused by the area reduction at the graft bifurcation.

\section{DISCUSSION}

Using a novel Y-graft baffle in computational flow dynamic simulations of Fontan hemodynamics, we have 

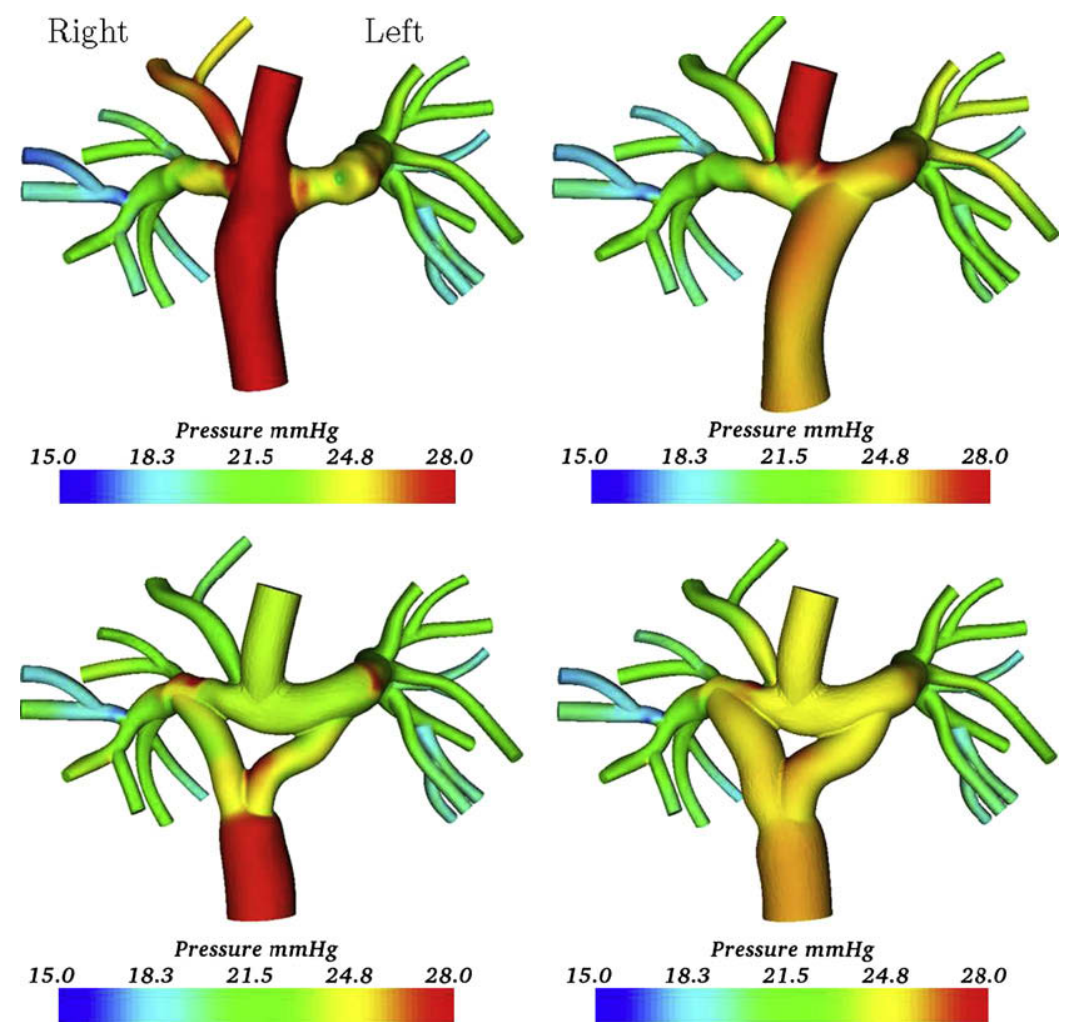

FIGURE 4. Contours of pressure ( $\mathrm{mm} \mathrm{Hg}$ ) from simulations of moderate exercise. Pressures shown correspond to the peak of inspiration when velocity is maximum.

demonstrated a significant improvement in Fontan performance. The large Y-graft design showed substantial improvement in pressure levels, efficiency, and flow distribution compared with the offset and t-junction designs used in current clinical practice. The relative rankings of the competing designs for efficiency, pressure level, and flow distribution are summarized in Table 4. WSS results were not conclusive enough to rank designs and are discussed at the end of this section. We also examined an RPA-offset model, and results with this model were similar to the LPA-offset case and therefore were not presented here.

Previous simulations of Fontan hemodynamics have usually focused on rest conditions and have reported very low energy dissipation values, leading to the belief among some that any gains in efficiency from geometric changes would be incremental at best. ${ }^{4,29,30}$ It is widely known, however, that during exercise, flow rates in the IVC increase considerably owing to the additional demand for blood in the lower part of the body, a consequence of leg muscle movement. ${ }^{23,31}$ Our previous work has demonstrated large decreases in efficiency and increases in pressure with increasing flow rates during simulated exercise. ${ }^{11}$ On the basis of these findings, we believe it is not sufficient to fully evaluate the performance of new Fontan designs without considering a range of rest and exercise conditions. We have taken this approach in evaluating the Y-graft design.
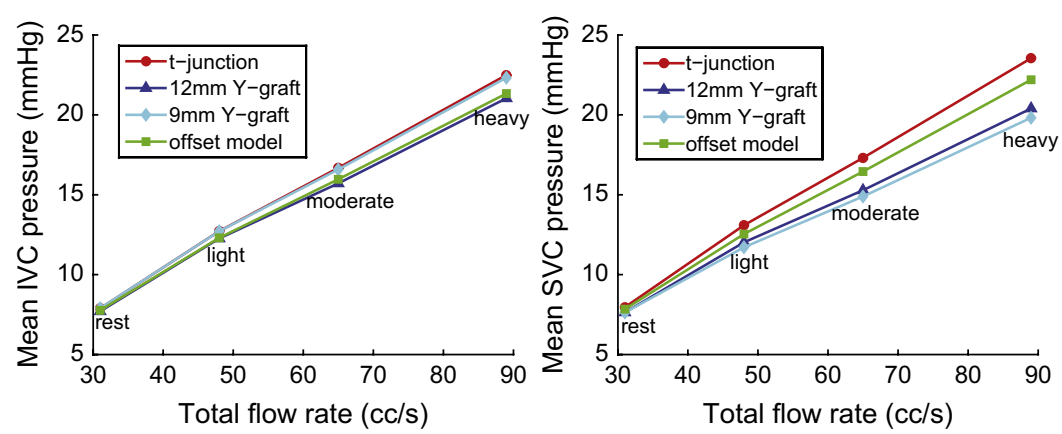

FIGURE 5. Simulation results for IVC (left) and SVC (right) pressures for each of the four Fontan designs. Pressure values (mm Hg) represent the mean value over one respiratory cycle. 


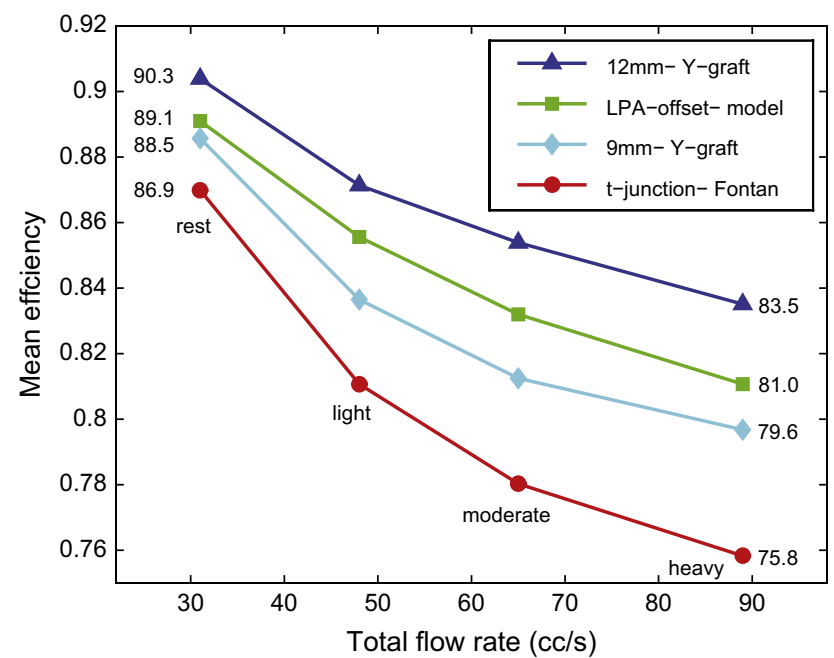

FIGURE 6. Comparison of energy efficiency of t-junction, offset, large-Y, and small-Y Fontan designs at rest, light, moderate, and heavy exercise. Efficiency values are mean over one respiratory cycle. $L P A$, Left pulmonary artery.

Additionally, most previous studies have used steady inflow conditions or have only included the cardiac pulsatility ${ }^{1-4,32}$ without considering respiration. In contrast, our respiratory model results in time-varying flow rates and pressures that are consistent with clinical observations. Both echocardiography tracings ${ }^{11}$ and real-time PC-MRI measurements ${ }^{31,33}$ have shown significant variation (as much as $80 \%$ ) in Fontan IVC flow rates with respiration. Similar variation is found in pressure tracings from cardiac catheterization, and previous work using the respiration model demonstrated good agreement with pressure tracings from catheterization. ${ }^{11}$ The respiration model is used in combination with sophisticated outflow boundary conditions that allow for physiologic levels of pressure. ${ }^{14}$ With these capabilities, our simulation results are better matched to clinically observed flow rates and pressures than was possible in previous Fontan simulations.

A recent study by Soerensen and associates ${ }^{13}$ proposed a new TCPC geometry called the "OptiFlo" that bifurcates both the IVC and SVC. Our proposed Y-graft design and their OptiFlo design share the concept of avoiding direct IVC/SVC flow competition while maintaining symmetry

TABLE 2. Distribution of IVC flow to the LPA and RPA during resting flow conditions

\begin{tabular}{lcc}
\hline Fontan design & Percent flow to LPA & Percent flow to RPA \\
\hline t-Junction & 53 & 47 \\
LPA offset model & 70 & 30 \\
Y-graft $(9 \mathrm{~mm})$ & 42 & 58 \\
Y-graft $(12 \mathrm{~mm})$ & 45 & 55 \\
\hline
\end{tabular}

$I V C$, Inferior vena cava; $L P A$, left pulmonary artery; $R P A$, right pulmonary artery. A particle-tracking method was used to advect particles from the IVC to the lungs to quantify flow distribution.
TABLE 3. Minimum and mean (in space) WSS values over the graft portion of the model only

\begin{tabular}{lcc}
\hline Fontan design & $\begin{array}{c}\text { Minimum WSS on } \\
\text { graft }\left(\mathbf{d y n e} / \mathbf{c m}^{\mathbf{2}}\right)\end{array}$ & $\begin{array}{c}\text { Mean WSS on } \\
\text { graft }\left(\mathbf{d y n e} / \mathbf{c m}^{\mathbf{2}}\right)\end{array}$ \\
\hline t-Junction & 0.15 & 3.6 \\
LPA offset model & 0.16 & 4.0 \\
Y-graft $(9 \mathrm{~mm})$ & 0.24 & 9.3 \\
Y-graft $(12 \mathrm{~mm})$ & 0.14 & 5.3 \\
\hline
\end{tabular}

WSS, Wall shear stress; $L P A$, left pulmonary artery. Values are the average over one respiratory cycle during resting flow conditions.

of the TCPC junction. In addition, our Y-graft design is very similar to one of the alternate designs they propose, in which only the IVC is bifurcated. Both the Y-graft and OptiFlow designs demonstrate increased energy efficiency compared with current offset designs. From a modeling standpoint, the Y-graft simulations presented here address some limitations of those reported by Soerensen's group, ${ }^{13}$ including simplified geometries and steady inflow conditions.

There are a few key differences that distinguish the Ygraft design from the OptiFlo and make it more practical from a surgical standpoint. First, the proposed Y-graft would be custom manufactured of synthetic material, following current surgical practice for the extracardiac conduit. ${ }^{9}$ As currently proposed by Soerensen and associates, ${ }^{13}$ the OptiFlo would be created by bifurcating the patient's native vessel in both the SVC and IVC. This makes the assumption that the native IVC vessel will reach the pulmonary arteries without requiring a synthetic graft. As a result, the OptiFlo graft dimensions are much smaller than currently used grafts because of the assumption that the IVC tissue will grow. An additional advantage of the Y-graft design is that it can be optimized or modified for an individual patient by custom manufacturing the graft portion before surgery.

It is likely that the Y-graft efficiencies could be improved further by bifurcating the SVC, as proposed in the OptiFlo design. However, this would require an increased level of surgical complexity with modifications to both the Glenn and Fontan procedures, introduction of synthetic material in the SVC, and significantly more suturing. In contrast, our proposed Y-graft Fontan can be implemented with

TABLE 4. Relative rankings of Fontan designs

\begin{tabular}{lcccc}
\hline Fontan design & Efficiency & $\begin{array}{c}\text { IVC } \\
\text { pressure }\end{array}$ & $\begin{array}{c}\text { SVC } \\
\text { pressure }\end{array}$ & $\begin{array}{c}\text { Flow } \\
\text { distribution }\end{array}$ \\
\hline t-Junction & $\sqrt{ }$ & $\sqrt{ }$ & $\sqrt{ }$ & $\sqrt{ }$ \\
LPA offset & $\sqrt{ } \sqrt{ }$ & $\sqrt{ } \sqrt{ }$ & $\sqrt{ }$ & $\sqrt{ }$ \\
Y-graft $(9 \mathrm{~mm})$ & $\sqrt{ }$ & $\sqrt{ }$ & $\sqrt{ } \sqrt{ }$ & $\sqrt{ } \sqrt{ }$ \\
Y-graft $(12 \mathrm{~mm})$ & $\sqrt{ } \sqrt{ } \sqrt{ }$ & $\sqrt{ } \sqrt{ } \sqrt{ }$ & $\sqrt{ } \sqrt{ }$ & $\sqrt{ } \sqrt{ } \sqrt{ }$ \\
\hline$I V C$, Inferior vena cava; $S V C$, superior vena cava; $L P A$, left pulmonary artery. The de- \\
signs are ranked $(\sqrt{ } \sqrt{ } \sqrt{ }=$ best, $\sqrt{ }=$ worst) according to performance for efficiency, \\
pressure level, and flow distribution. The 12-mm Y-graft is the best overall performing \\
on the basis of these criteria.
\end{tabular}


a pre-made IVC graft, does not interfere with the native SVC, and allows for the operation to be performed without cardiopulmonary bypass. ${ }^{9}$ To make a thorough comparison, analysis of the OptiFlo (which used cylindrical models and steady flow simulations) should be extended to include patient-specific geometries (including representative branches of the pulmonary arteries) and physiologic flow and pressure conditions in future work. Further optimization on the Ygraft should be performed, for example, using the methods presented by Marsden, Feinstein, and Taylor ${ }^{34}$ for optimization of cardiovascular geometries.

Pressure levels in the IVC and SVC are important measures of Fontan performance and are known to be important determinants of long-term outcomes, with elevated pressures often linked with poor outcomes. Pressure levels in both the IVC and SVC were significantly reduced with the new Y-graft design, even in the setting of presumed exceptional hemodynamics given the catheterization measured mean pressures of $7 \mathrm{~mm} \mathrm{Hg}$. The difference was particularly pronounced during exercise. The long-term effects of elevated IVC and SVC pressures on exercise intolerance and Fontan outcomes are not fully understood. Low Fontan pressures are generally indicative of better clinical outcomes, albeit far from any guarantee of positive long-term outcomes. The Y-graft design also offers the advantage that SVC pressure is decoupled from the IVC pressure so that SVC pressure can remain lower than IVC pressure during exercise. This effect was demonstrated by our exercise simulations.

These simulations also show a significant improvement in energy efficiency in the large Y-graft model when compared with the other models. Previous work by Marsden and colleagues ${ }^{11}$ has also demonstrated large differences in efficiency between patients with different Fontan geometries. Clinical studies will be needed to correlate improvements in efficiency with differences in exercise tolerance and long-term outcomes in Fontan patients. However, it is logical to assume that any increase in efficiency should, in theory, reduce the work load on the system and be beneficial to the patient. Although efficiency improvements of only a few percentages may initially seem insignificant, differences of only a few percentages are similar to the effects one might see with a doubling of the IVC flow rate from rest to exercise. This leads us to believe these differences do, in fact, have potential to be significant for improving exercise capacity for these patients.

Previous studies have shown that a yet undetermined hepatic factor is essential for normal lung development and that the lack of this factor can lead to the development of arteriovenous malformations. ${ }^{35,36}$ The distribution of IVC flow will affect the concentration of hepatic factor present in the lungs. Although it is not known what concentration is required for normal lung development, if all other factors are equal, it is clearly desirable to distribute the flow (and, additionally, the relative "work'" required by each lung) as evenly as possible. The proposed large-Y design distributes the IVC flow according to the $45 / 55$ flow split. In contrast, we have found that the distribution of IVC flow with the offset design is strongly skewed toward the side of the offset. The IVC flow distribution in the large-Y connection appears relatively robust to local asymmetries; however, the t-junction and small-Y geometries appear more sensitive to asymmetries, which result in moderate changes from the 45/55 distribution.

One theoretical concern raised by the Y-graft results is the potential for thrombus formation resulting from flow stasis. Previous studies have shown that anticoagulation (with either aspirin or warfarin sodium) improves outcomes postoperatively for Fontan patients and that the incidence of thrombus formation at varying locations is $10 \%$ to $20 \%$, regardless of the type of Fontan surgery. ${ }^{37,38}$

Our results show that the Y-graft model has slightly larger areas of low shear, but that the minimum WSS values are similar in value to other designs. It is not currently known what minimum levels of shear or particle residence time are needed to prevent thrombosis. The areas of low WSS areas observed in the Y-graft at rest should be investigated further to evaluate this risk. Because the low shear areas are coincident with areas of high local curvature in the model, we believe it is likely that optimization of the Y-graft geometry could eliminate local areas of low shear while maintaining higher efficiency. This will be investigated in future work on Y-graft optimization, in which a more gradual tapering from trunk to branches could be implemented. An additional concern could be the development of hypoplasia or even discontinuity between the two limbs of the Y-graft. This also should be investigated further. However, the results presented here do not indicate any areas of low WSS in this region (Figure E2).

Technical limitations of the $\mathrm{Y}$-graft procedure compared with current surgical techniques include the need for more suturing (and suture lines) and an increased level of complexity in performing the operation. Integration of the model into a volume rendering of the MRI data from the patient shows no compression of the pulmonary veins or other barriers to technical feasibility. We also note that the Y-graft design should not impose anatomic space requirements beyond what is already routinely done in either a right or left offset procedure with a larger diameter baffle. A potential advantage from a technical standpoint is that the Y-graft could be clamped on the left side during surgery to patch the LPA in cases of LPA stenosis.

\section{LIMITATIONS}

These simulations represent a single patient model. Additional studies extending this work to multiple patients to verify improvements in efficiencies are required. We note that our definition of Fontan efficiency considers the energy dissipation of the Fontan geometry alone, without modeling the entire single ventricle circulation. Previous studies have 
considered alternate normalization strategies for the energy dissipation. ${ }^{12}$ Coupling Fontan simulations to a single-ventricle heart model to model the energetics of the entire circulatory system could be an interesting area for future investigation. Additionally, patient-specific data with exercise is needed to validate our results (and will increase the accuracy of future simulations).

An additional limitation of this work is the use of rigid walls in our simulations. It is expected that adding vessel compliance to the model will slightly decrease the efficiencies we have reported because of added energy dissipation. However, we do not expect this to change the relative performance of the four Fontan designs considered in this work.

There is also a need for more data on pulmonary arterial physiology and pulmonary flow distribution in Fontan patients. In particular, the extent to which the distal pulmonary arteries of Fontan patients will dilate during exercise is unknown. In this study, resistance was reduced somewhat less than that of healthy children on the basis of the assumption that Fontan patients are, at baseline, vasodilated and have limited capacity for further pulmonary vasodilation. Additionally, further data are needed to better quantify the distribution of flow among the lung segments. When available, these data should be incorporated into future Fontan modeling studies.

\section{CONCLUSIONS}

Using computational blood flow simulations and patientspecific models, we have demonstrated that our proposed Ygraft modification of the Fontan operation results in higher hemodynamic efficiency during rest and exercise compared with designs used in current clinical practice. In addition, our simulations predict lower Fontan pressures using the Y-graft design, particularly at exercise. It is widely known that lower Fontan pressures correlate with improved long-term outcomes in Fontan patients. IVC flow distribution is also significantly improved with the proposed Y-graft design. The increased efficiency, lower pressures, and improved hepatic factor distribution observed in our results suggest that the Ygraft could be a promising modification of the Fontan procedure. It is our hope that this new design will lead to improved exercise tolerance and overall outcomes for these patients. This work also demonstrates the role that simulations can play in predicting the performance of new surgical designs at no risk to the patient. Clinical trials are needed to determine the clinical benefits of the Y-graft procedure compared with current surgical practice.

\section{References}

1. Ryu K, Healy TM, Ensley AE, Sharma S, Lucas C, Yoganathan AP. Importance of accurate geometry in the study of the total cavopulmonary connection: computational simulations and in vitro experiments. Ann Biomed Eng. 2001;29:844-53.

2. Khunatorn Y, Mahalingam S, DeGroff CG, Shandas R. Influence of connection geometry and SVC-IVC flow rate ratio on flow structures within the total cavopulmonary connection: a numerical study. J Biomech Eng. 2002;124:364-77.
3. Migliavacca F, Dubini G, Bove EL, de Leval MR. Computational fluid dynamics simulations in realistic 3-D geometries of the total cavopulmonary anastomosis: the influence of the inferior caval anastomosis. J Biomech Eng. 2003; 125:805-13.

4. Dubini G, de Leval MR, Pietrabissa R, Montevecchi FM, Fumero R. A numerical fluid mechanical study of repaired congenital heart defects: Application to the total cavopulmonary connection. J Biomech. 1996;29:111-21.

5. Bove EL, de Leval MR, Migliavacca F, Balossino R, Dubini G. Toward optimal hemodynamics: computer modeling of the Fontan circuit. Pediatr Cardiol. 2007; 28:477-81.

6. Ensley AE, Lynch P, Chatzimavroudis GP, Lucas C, Sharma S, Yoganathan AP. Toward designing the optimal total cavopulmonary connection: an in vitro study. Ann Thorac Surg. 2000;68:1384-90.

7. Gerdes A, Kinze J, Pfister G, Sievers HH. Addition of a small curvature reduces power losses across total cavopulmonary connections. Ann Thorac Surg. 1999; 67:1760-4.

8. Migliavacca F, Dubini G, Pietrabissa R, de Leval MR. Computational transient simulations with varying degree and shape of pulmonic stenosis in models of the bidirectional cavopulmonary anastomosis. Med Eng Phys. 1997;19:394-403.

9. Petrossian E, Reddy VM, Collins KK, Culbertson CB, MacDonald MJ, Lamberti JJ, et al. The extracardiac conduit Fontan operation using minimal approach extracorporeal circulation: early and midterm outcomes. J Thorac Cardiovasc Surg. 2006;1321054-63.

10. de Leval MR, Dubini G, Migliavacca F, Jalali H, Camporini G, Redington A, et al. Use of computational fluid dynamics in the design of surgical procedures: application to the study of competitive flows in cavopulmonary connections. J Thorac Cardiovasc Surg. 1996;111:502-13.

11. Marsden AL, Vignon-Clementel IE, Chan F, Feinstein JA, Taylor CA. Effects of exercise and respiration on hemodynamic efficiency in CFD simulations of the total cavopulmonary connection. Ann Biomed Eng. 2007;35:250-3.

12. Whitehead KK, Pekkan K, Kitahima HD, Paridon SM, Yoganathan AP, Fogel MA. Nonlinear power loss during exercise in single-ventricle patients after the Fontan: insights from computational fluid dynamics. Circulation. 2007;116(11 Suppl): I165-71.

13. Soerensen DD, Pekkan K, de Zelicourt D, Sharma S, Kanter K, Fogel M, et al. Introduction of a new optimized total cavopulmonary connection. Ann Thorac Surg. 2007;83:2182-90.

14. Vignon-Clementel IE, Figueroa CA, Jansen KE, Taylor CA. Outflow boundary conditions for three-dimensional finite element modeling of blood flow and pressure in arteries. Comput Methods Appl Mech Eng. 2006;195:3776-96.

15. Spilker RL, Feinstein JA, Parker DW, Reddy VM, Taylor CA. Morphometry based impedance boundary conditions for patient-specific modeling of blood flow in pulmonary arteries. Ann Biomed Eng. 2007;35:546-9.

16. Figueroa CA, Vignon-Clementel IE, Jansen KE, Hughes TJR, Taylor CA. A coupled momentum method for modeling blood flow in three- dimensional deformable arteries. Comput Methods Appl Mech Eng. 2006;195:5685-706.

17. Muller J, Sahni O, Li X, Jansen KE, Shephard MS, Taylor CA. Anisotropic adaptive finite element method for modelling blood flow. Comput Methods Biomech Biomed Eng. 2005;8:295-305.

18. Taylor CA, Draney MT, Ku JP, Parker D, Steele BN, Wang K, et al. Predictive medicine: computational techniques in therapeutic decision-making. Comput Aid Surg. 1999;4:231-47.

19. Wilson N, Wang K, Dutton R, Taylor CA. A software framework for creating patient specific geometric models from medical imaging data for simulation based medical planning of vascular surgery. Lect Notes Comput Sci. 2001;2208: 449-56.

20. Alexi-Meskishvili V, Ovroutski S, Ewert P, Dahnert I, Berger F, Lange PE, et al. Optimal conduit size for extracardiac Fontan operation. Eur J Cardiothorac Surg. 2000; 18:690-5.

21. Taylor CA, Hughes TJR, Zarins CK. Finite element modeling of blood flow in arteries. Comput Method Appl Mech Eng. 1998;158:155-96.

22. Whiting CH, Jansen KE. A stabilized finite element method for the incompressible Navier-Stokes equations using a hierarchical basis. Int J Num Methods Fluids. 2001;35:93-116.

23. Cheng CP, Herfkens RJ, Lightner AL, Taylor CA, Feinstein JA. Blood flow conditions in the proximal pulmonary arteries and vena cavae: healthy children during upright cycling exercise. Am J Physiol Heart Circ. 2004;287:921-6.

24. Kulik TJ, Bass JL, Fuhrman BP, Moller JH, Lock JE. Exercise induced pulmonary vasoconstriction. Br Heart J. 1983;50:59-64.

25. Westerhof N, Bosman F, De Vries CJ, Noordergraaf A. Analog studies of the human systemic arterial tree. J Biomech. 1969;2:121-43. 
26. Grigioni M, D'Avenio G, Amodeo A, Di Donato RM. Power dissipation associated with surgical operations' hemodynamics: critical issues and application to the total cavopulmonary connection. J Biomech. 2006;39:1583-94.

27. Shadden SC, Taylor CA. Characterization of coherent structures in the cardiovascular system. Ann Biomed Eng. 2008;36:1152-62.

28. Fehlberg E. Low-order classical runge-kutta formulas with step size control and their application to some heat transfer problems. Rep. NASA TR R-315, George C. Marshall Space Flight Center, Marshall, Ala., 1969.

29. Moyle KR, Mallinson GD, Occleshaw CJ, Cowan BR, Gentles TL. Wall shear stress is the primary mechanism of energy loss in the Fontan connection. Pediatr Cardiol. 2006;27:309-15.

30. Healy TM, Lucas C, Yoganathan AP. Noninvasive fluid dynamic power loss assessments for total cavopulmonary connections using the viscous dissipation function: a feasibility study. J Biomech Eng. 2001;123:317-24.

31. Hjortdal VE, Emmertsen K, Stenbog E, Frund T, Rahbek Schmidt M, Kromann O, et al. Effects of exercise and respiration on blood flow in total cavopulmonary connection: a real-time magnetic resonance flow study. Circulation. 2003;108:1227-31.

32. Migliavacca F, Kilner PJ, Pennati G, Dubini G, Pietrabissa R, Fumero R, et al. Computational fluid dynamic and magnetic resonance analyses of flow distribu- tion between the lungs after total cavopulmonary connection. IEEE Trans Biomed. Eng. 1999;46:393-9.

33. Hsia T, Khambadkone S, Redington AN, Migliavacca F, Deanfield JE, de Leval MR. Effects of respiration and gravity on infradiaphragmatic venous flow in normal and Fontan patients. Circulation. 2000;102(Suppl III):III148-53.

34. Marsden AL, Feinstein JA, Taylor CA. A computational framework for derivative- free optimization of cardiovascular geometries. Comput Methods Appl Mech Eng. 2008;197:1890-905.

35. Justino H, Benson LN, Freedom RM. Development of unilateral pulmonary arteriovenous malformations due to unequal distribution of hepatic venous flow. Circulation. 2001;103:E39-40.

36. Pike NA, Vricella LA, Feinstein JA, Black MD, Reitz BA. Regression of severe pulmonary arteriovenous malformations after Fontan revision and hepatic factor rerouting. Ann Thorac Surg. 2004;78:697-9.

37. Coon PD, Rychik J, Novello RT, Ro PS, Gaynor JW, Spray TL. Thrombus formation after the Fontan operation. Ann Thorac Surg. 2001;71:1990-4.

38. Rosenthal DN, Friedman AH, Kleinman CS, Kopf GS, Rosenfeld LE, Hellenbrand WE. Thromboembolic complications after Fontan operations. Circulation. 1995;92:287-93. 

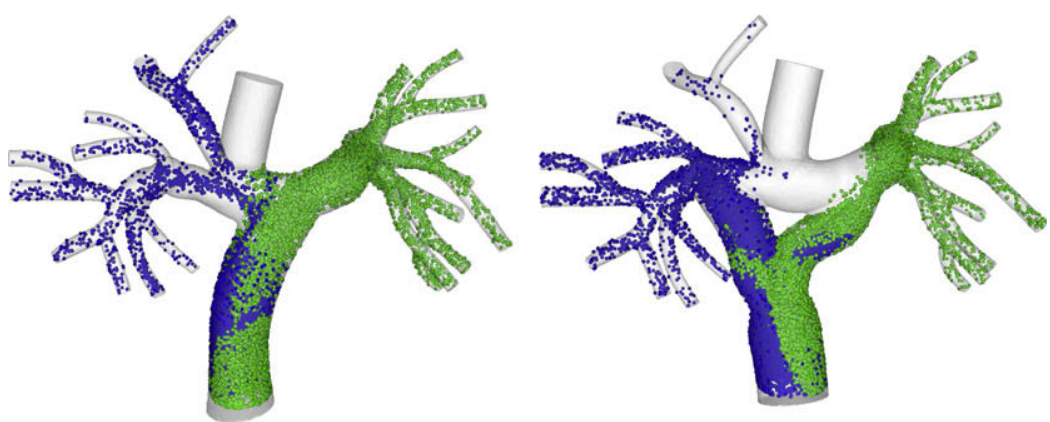

FIGURE E1. Illustration of the particle-tracking method used to quantify the proportion of IVC flow to the LPA and RPA. Particles are injected in the IVC and advected with the flow. Blue particles travel to the RPA side and green particles travel to the LPA side. $L P A$, left pulmonary artery; RPA, right pulmonary artery. 

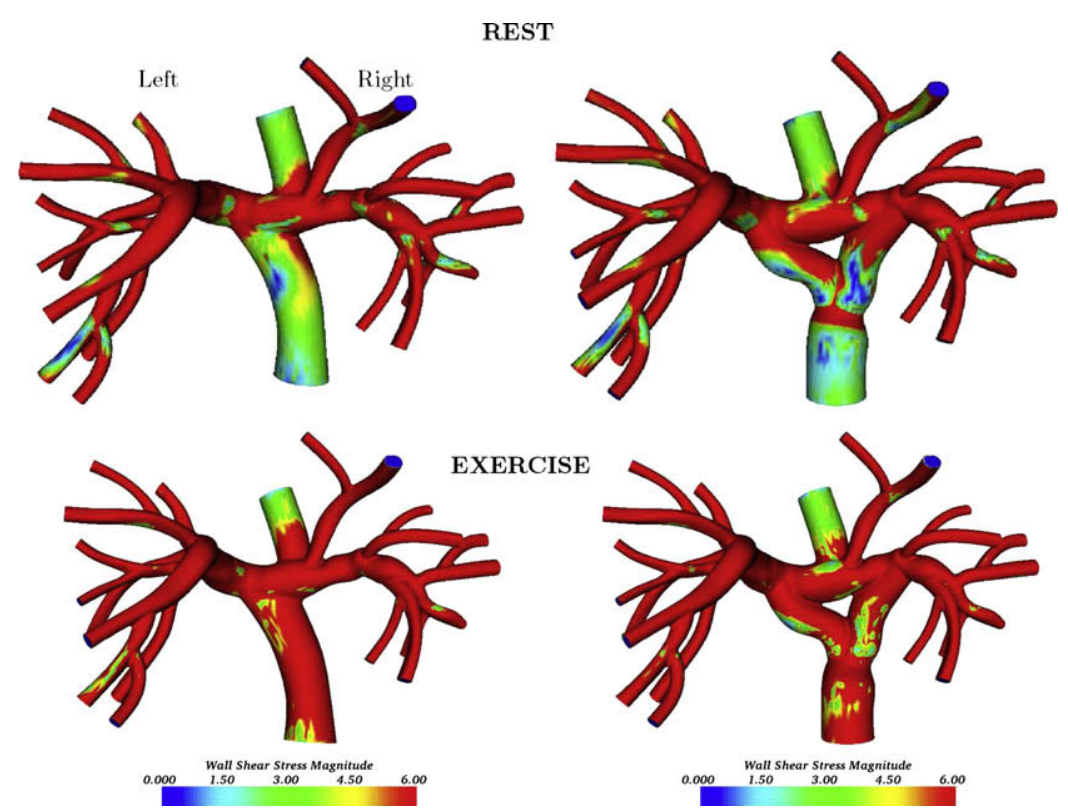

FIGURE E2. Comparison of mean wall shear stress $\left(\right.$ dynes $/ \mathrm{cm}^{2}$ ) over one respiratory cycle for the offset model (left) and large Y-graft model (right) during resting (top row) and moderate exercise (bottom row) flow conditions. Models are rotated for better viewing. 